Classification
Physics Abstracts
$68.10-64.75-64.70 \mathrm{~J}-36.20$

\title{
INTERFACE IN MOLTEN POLYMER MIXTURES NEAR THE CONSOLUTE POINT
}

\author{
J. F. JOANNY and L. LEIBLER $\left(^{*}\right)$ \\ Collège de France, 75231 Paris Cedex 05, France
}

(Reçu le 6 mars 1978, accepté le 1er juin 1978)

\begin{abstract}
Résumé. - On étudie dans le cadre d'une théorie de champ moyen les propriétés interfaciales d'un système de polymères fondus à une température inférieure à la température critique de démixtion mais voisine de celle-ci. La nature de cet interface est très particulière, il est beaucoup plus large que dans le cas usuel des mélanges de liquides et la tension interfaciale est beaucoup plus petite. Seules des méthodes optiques devraient permettre de l'étudier expérimentalement.
\end{abstract}

Abstract. - The interfacial properties of a system of molten polymers at a temperature just below the critical temperature of demixing are studied in the framework of a mean field theory. The properties of the interface are very specific, the interface is much larger than those of usual binary liquids and the interfacial tension is much smaller so that only optical methods will permit experimental study.

1. Introduction. - In recent years much work has been devoted to the study of heterogeneous macromolecular systems and in particular of the interfaces (see, e.g. the review article by Helfand [1] and references therein). The most characteristic feature of the twocomponent polymeric system is a strong repulsion between unlike molecules even when the repulsion between unlike monomers is relatively small. As a result most pairs of polymeric substances are immiscible in each other, i.e. their consolute point temperatures are extremely high [2]. The nature of the interfacial region between two polymer phases depends crucially on the degree of incompatibility. Till now there has been much concern with theoretical description of the interface far from the critical point of demixing (see, e.g. [3-6]) and a reasonable agreement with the measurements of the interfacial tension $[7,8]$ has been found [5].

In the present paper we shall study the properties of the interface very near the critical point of demixing (consolute point). This problem seems to be of interest for various reasons : $a$ ) The study of the interfacial region in binary liquids near the consolute point provides a direct method of measuring the correlation

$\left({ }^{*}\right)$ On leave of absence from Institute of Theoretical Physics, Warsaw University, 00-681 Warsaw, Poland. length $\xi$ and the corresponding critical exponent $v$ [9]; b) The behaviour of polymeric substances is very different from those of binary mixtures of small molecules. The thickness of the interface which is of the order of correlation length $\xi$ is much larger and the interfacial tension $\gamma_{\mathbf{s}}$ turns out to be much smaller; c) From the point of view of the theory of phase transitions binary liquids play an important role : they fall in the same universality class as the gas-liquid transition and the static critical exponents should be those of Ising model. However for molten polymers the critical point of demixing is expected to be well described by the mean field theory of Flory and Huggins (see $[10,11,15])$. Thus, the interface properties may be described by a simple theory and the measurements of the interfacial length may serve as an experimental test of the validity of the theory. It should be stressed however that when the system composed of a polymer liquid and a solvent is considered the fluctuations play an important role, and the Flory-Huggins theory fails [10]. Therefore, the results of reference [16] for interfacial properties of a polymer solution near the consolute point obtained in the framework of a meanfield theory may serve only as a first approximation.

In section 2 we determine the concentration profile and the interfacial tension in a system of two molten polymers at the critical concentration and just below the critical temperature. Possible experimental 
methods of determining the interfacial properties are discussed in section 3 .

2. Interfacial properties. - We consider a system composed of two molten polymers $\mathrm{A}$ and $\mathrm{B}$ with polymerization indices $N_{\mathrm{A}}$ and ${ }_{\mathrm{B}}$, respectively. The concentration of monomers $\mathrm{A}$ and $\mathrm{B}$ will be denoted by $\rho_{\mathrm{A}}=\rho$ and $\rho_{\mathrm{B}}=1-\rho$. (We assume that the system is incompressible and to avoid cumbersome factors we take the monomer length a as the unit of length.)

The free-energy per unit volume of such a system is given by $[10,11]$

$$
F=k T\left(\frac{\rho_{\mathrm{A}}}{N_{\mathrm{A}}} \ln \rho_{\mathrm{A}}+\frac{\rho_{\mathrm{B}}}{N_{\mathrm{B}}} \ln \rho_{\mathrm{B}}\right)+u \rho_{\mathrm{A}} \rho_{\mathrm{B}}
$$

where $u$ is the interaction energy between monomers A and $\mathrm{B}$ related to the Flory parameter $\chi_{\mathrm{F}}$ by $u=k T \chi_{\mathrm{F}}$.

For the temperature above the critical temperature of demixing $T_{\mathrm{c}}$ the polymers are miscible in all proportions. The critical point of demixing is determined by the relation

$$
k T_{\mathrm{c}}=\frac{2 u N_{\mathrm{A}} N_{\mathrm{B}}}{\left(N_{\mathrm{A}}^{1 / 2}+N_{\mathrm{B}}^{1 / 2}\right)^{2}}, \quad \rho_{\mathrm{c}}=\frac{N_{\mathrm{B}}^{1 / 2}}{N_{\mathrm{A}}^{1 / 2}+N_{\mathrm{B}}^{1 / 2}} .
$$

We shall be concerned with the system at the temperature just below $T_{\mathrm{c}}$, i.e. $-\varepsilon \equiv\left(T_{\mathrm{c}}-T\right) / T_{\mathrm{c}} \ll 1$, and with the overall concentration of $\mathrm{A}$ equal to $\rho_{\mathrm{c}}$. In such a system two phases are in equilibrium. The existing self-consistent theories $[3,5,6]$ of the interface between two polymers phases do not incorporate the specific features of the critical region (and in particular the large thickness of the transition region).

As $|\varepsilon| \ll 1$ the difference $\delta \rho$ between concentration in the separated phases $\rho$ and the critical concentration $\rho_{\mathrm{c}}$ is supposed to be small so that the free energy $F$ of the system may be expanded in powers of $\delta \rho$ (for small $\delta \rho$ )

$$
F / k T=1 / 2 B \delta \rho^{2}+1 / 4 C \delta \rho^{4} .
$$

The third derivative of the free energy vanishes for $\rho=\rho_{\mathrm{c}}$ even when $T \neq T_{\mathrm{c}}$. It is due to a specific form of Flory interaction,

and

$$
B=\varepsilon\left[\frac{1}{\rho_{\mathrm{c}} N_{\mathrm{A}}}+\frac{1}{\left(1-\rho_{\mathrm{c}}\right) N_{\mathrm{B}}}\right]
$$

$$
C=1 / 3\left[\frac{1}{N_{\mathrm{A}} \rho_{\mathrm{c}}^{3}}+\frac{1}{N_{\mathrm{B}}\left(1-\rho_{\mathrm{c}}\right)^{3}}\right]
$$

(such an expression for the free energy is valid only near the critical point).

The concentration of monomers $\mathrm{A}$ in the two phases is determined by the condition that $F$ is minimum, which gives

$$
C \delta \rho^{2}+B=0 \text {. }
$$

We shall denote by $\delta \rho_{\infty}$ and $-\delta \rho_{\infty}$ the two solutions of equation (6).

However, the assumption that $\delta \rho$ is constant is a simplification; spatial variation of $\delta \rho$ must be taken into account. Let us suppose that the interface is perpendicular to $x$-axis and that $\delta \rho$ changes only along $x$-axis; when $x \rightarrow+\infty$

$$
\delta \rho=\delta \rho_{\infty}
$$

and when $x \rightarrow-\infty$

$$
\delta \rho=-\delta \rho_{\infty}
$$

When $\rho$ changes in space the free energy may be approximated by

$F=1 / 2 \sum_{q} \chi^{-1}(q)|\delta \rho(q)|^{2}+$ terms of the order $\delta \rho^{4}$

$\delta \rho(q)$ - denotes the Fourier transform of $\delta \rho(x)$.

$\chi(q)$ is the osmotic compressibility of the system given by [11]

$$
\chi^{-1}(q)=B\left(1+q^{2} \xi^{2}\right)
$$

where we have introduced the correlation length $\xi$ (see $[11,15])$

$$
\xi=\left[1 / 18\left(\frac{1}{\rho_{\mathrm{c}}}+\frac{1}{1-\rho_{\mathrm{c}}}\right)\right]^{1 / 2}|B|^{-1 / 2} .
$$

Hence, the Landau free energy density reads

$$
\frac{F}{k T}=\frac{B}{2} \delta \rho^{2}+1 / 2 B \xi^{2}|\nabla \delta \rho|^{2}+1 / 4 C \delta \rho^{4}
$$

To determine the concentration profile at equilibrium the free energy $F$ should be minimized. The LagrangeEuler equation [12]

$$
\frac{\partial F}{\partial \delta \rho}=\frac{\partial}{\partial x} \frac{\partial F}{\partial(\partial \delta \rho / \partial x)}
$$

leads to the differential equation for $\delta \rho(x)$

$$
B \xi^{2} \frac{\mathrm{d}^{2} \delta \rho}{\mathrm{d} x^{2}}=+B \delta \rho+C \delta \rho^{3}
$$

The solution which satisfies the boundary conditions imposed at $x \rightarrow \pm \infty$ reads

$$
\delta \rho=\delta \rho_{\infty} \text { th }\left(\frac{x}{\sqrt{2} \xi}\right) .
$$

The plot of the concentration profile through the interface is given on figure 1 . The thickness $L$ of the interface given by

$$
L=\sqrt{2} \xi
$$

is of the order of the correlation length as might be expected. 


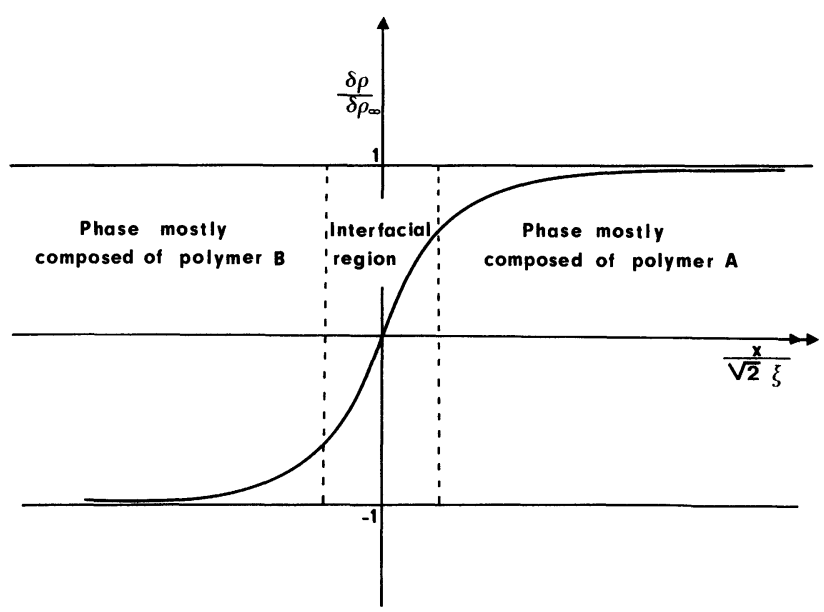

FIG. 1. - Concentration profile in the interfacial region.

The interfacial tension is defined by (see e.g. [13])

$$
\gamma_{\mathrm{s}}=\int_{-\infty}^{\infty} \mathrm{d} x\left[F(\delta \rho)-F\left(\delta \rho_{\infty}\right)\right] .
$$

Making use of equations (10), (6) and (13) we get

$$
\begin{aligned}
\gamma_{\mathrm{S}} & =\frac{2}{3}\left\{|\varepsilon| \frac{1}{N_{\mathrm{A}} \rho_{\mathrm{c}}}+\frac{1}{N_{\mathrm{B}}\left(1-\rho_{\mathrm{c}}\right)} \mid\right\}^{3 / 2} \times \\
& \times\left(\frac{1}{\rho_{\mathrm{c}}}+\frac{1}{1-\rho_{\mathrm{c}}}\right)^{1 / 2}\left[\frac{1}{N_{\mathrm{A}} \rho_{\mathrm{c}}^{3}}+\frac{1}{N_{\mathrm{B}}\left(1-\rho_{\mathrm{c}}\right)^{3}}\right]^{-1} .
\end{aligned}
$$

43. Discussion. - We shall discuss in detail the symmetric case, i.e. systems with $N_{\mathrm{A}}=N_{\mathrm{B}}=N$. In such a case $\rho_{\mathrm{c}}=1 / 2$ and from (9) and (16) we get

$$
\begin{aligned}
& L=1 / 3|\varepsilon|^{-1 / 2} a N^{1 / 2} \\
& \gamma_{\mathrm{s}}=2 / 3 k T \frac{|\varepsilon|^{3 / 2}}{N^{1 / 2} a^{2}}
\end{aligned}
$$

where we have introduced $a$ the unit of the statistical length of the two polymers.

At this point an interesting remark may be made. Comparing (17) and (18) we see that $\gamma_{\mathrm{S}}$ is not proportional to $k T / \xi^{2}$ as might be expected. Actually the scaling laws for critical phenomena in systems with strong fluctuations would predict a free energy $F \sim|\varepsilon|^{2-\alpha}$ and an interfacial tension

$$
\gamma_{\mathrm{s}} \sim F \xi \sim|\varepsilon|^{2-\alpha-v}
$$

in an Ising model

$$
v d=2-\alpha
$$

( $d$ - space dimension, $\alpha$ - the specific heat critical exponent) so that $\gamma_{S} \sim \varepsilon^{v(d-1)}$ which is $k T / \xi^{2}$ for $d=3$. But the scaling relation (19) is not verified by the mean field exponents (except for dimension 4) so $\gamma_{\mathrm{s}}$ is not proportional to $k T / \xi^{2}$.

Equations (17) and (18) permit estimation of the magnitude of both the interfacial tension and penetration depth. It should be stressed that in the vicinity of the consolute point the values of both $\xi$ and $\gamma_{\mathrm{S}}$ differ essentially from those far from the critical point. Because of the factor $N^{1 / 2}$ the interface length is much bigger than usual whereas the surface tension is much smaller. In fact for a of the order of few angströms, $N^{1 / 2} \sim 50-100, T \sim 100 \mathrm{~K}$ and $|\varepsilon| \sim 10^{-2}$ we find $\xi \sim 10^{3} \AA$ and $\gamma_{\mathrm{S}} \sim 10^{-4}$ dyne/cm, i.e. about 100 times smaller than the interfacial tension of small molecule binary liquids for the same $\varepsilon$, and $10^{4}$ times smaller than the interfacial tension of nearly compatible polymeric mixtures far from the consolute point.

There are only few polymer pairs [2] which show a consolute point temperature enabling experiments in the critical region. Examples of such systems are polystyrene- $\alpha$-methyl styrene and polybutadiene-polydimethyl siloxane.

Near the critical point $\gamma_{\mathbf{s}}$ is very small so that mechanical measurements do not seem to be appropriate. However, it may be expected that the optical methods of measuring $\gamma_{\mathrm{s}}$ and $L$ are quite suitable (see [9]). The measurement of reflectivity coefficient, which depends only on the thickness of interface $L$ and the equilibrium concentration $\delta \rho_{\infty}$, would provide a direct method of determining the correlation length $\xi$ and exponent $v$ (dependence of $\xi$ on $N$ ). It is also possible to determine $\gamma_{\mathbf{s}}$ by inelastic scattering of light [9]. However, as $\gamma$ is very small such an experiment seems to be difficult. The lowest interfacial tensions being measured by this method are of the order of $10^{-4}$ dyne/cm so one could not approach very near to the critical point [14].

Acknowledgments. - We are deeply indebted to Pr. P. G. de Gennes, Dr. D. Langevin and Dr. F. Rondelez for very helpful and interesting discussions.

\section{References}

[1] Helfand, E., Acc. Chem. Res. 8 (1975) 295.

[2] Krause, S., J. Macromol. Sci., Rev. Macromol. Chem. C7 (2) (1972) 251

[3] Helfand, E. and Tagami, Y., J. Polym. Sci. B 9 (1971) 741 ; J. Chem. Phys. 56 (1971) 3592; ibid. 57 (1972) 1812.

[4] Roe, R.-J., J. Chem. Phys. 60 (1974) 4192 ; ibid. 62 (1975) 490.

[5] Helfand, E. and SaPSe, A. M., J. Chem. Phys. 62 (1975) 1327.

[6] Helfand, E., J. Chem. Phys. 63 (1975) 2192.

[7] RoE, R. J., J. Colloid Interface Sci. 31 (1969) 228.

[8] Wu, S., J. Phys. Chem. 74 (1970) 632.

[9] Huang, J. S. and Webi, W. W., J. Chem. Phys. 50 (1969) 3677.
[10] De Gennes, P. G., J. Physique Lett. 38L (1977) 441.

[11] Joanny, J. F., C. R. Acad. Sci. 286 (1978) 89.

[12] Landau, L. D. and LifChitz, E., "Classical theory of fields " (Pergamon Press, London) 1975.

[13] Landau, L. D. and Lifchitz, E., "Statistical Physics » (Pergamon Press, London) 1958.

[14] Wu, E. S. and WeBs, W. W., J. Physique Colloq. 33 (1972) C1-149.

[15] Joanny, J. F., to be published in J. Phys. A.

[16] VRiJ, A., Roebersen, G. J., Journal of Polymer Science (Polymer Physics Edition) 15 (1977) 109. 